\title{
Internationale und deutsche Leitlinien zur Behandlung der PTBS bei Kindern und Jugendlichen
}

\section{Ein Überblick über aktuelle Empfehlungen}

\author{
Regina Steil ${ }^{1}$, Anne Fischer ${ }^{1}$ und Rita Rosner ${ }^{2}$ \\ 1Abteilung Klinische Psychologie und Psychotherapie, Goethe-Universität Frankfurt am Main \\ ${ }^{2}$ Lehrstuhl Klinische und Biologische Psychologie, Katholische Universität Eichstätt-Ingolstadt
}

\begin{abstract}
Zusammenfassung: Über die Hälfte aller Kinder und Jugendlichen erleben vor ihrem 18. Geburtstag ein potentiell traumatisierendes Ereignis. Entwickeln die Betroffenen in der Folge eine posttraumatische Belastungsstörung (PTBS), wirken sich die damit verbundenen Konsequenzen häufig bis ins Erwachsenenalter aus. Klinische Leitlinien unterstützen die Behandlerinnen und Behandler bei der Auswahl geeigneter Therapien, sind aufgrund variierender Methodik und unterschiedlicher Evidenzgrundlage aber mit Interpretationsschwierigkeiten verbunden. Dieser Beitrag gibt einen Überblick über aktuelle nationale und internationale Leitlinienempfehlungen. Als Methode erster Wahl zur Behandlung der PTBS bei Kindern und Jugendlichen wird übereinstimmend Psychotherapie in Form von Traumafokussierter Kognitiver Verhaltenstherapie (Tf-KVT) und teilweise auch Eye Movement Desensitization and Reprocessing (EMDR) empfohlen. Bezugspersonen sollen, wenn möglich, in die Behandlung einbezogen werden. Eine Pharmakotherapie wird nicht empfohlen. Forschungsbedarf besteht in den Bereichen der Dissemination empirisch gestützter Behandlungen, EMDR, und der Behandlung komplexer PTBS.
\end{abstract}

Schlüsselwörter: PTBS, Trauma, Kinder, Jugendliche, Behandlung, klinische Leitlinien

International and German Guidelines for the Treatment of PTSD in Children and Adolescents

Abstract: More than half of all children and adolescents experience a potentially traumatizing event before their $18^{\text {th }}$ birthday. If they subsequently develop a posttraumatic stress disorder (PTSD), the associated consequences may often continue into adulthood. Clinical treatment guidelines assist treatment providers in selecting appropriate therapies but are also linked with interpretive difficulties because of varying methodology and evidence base. This article provides an overview of current recommendations from international and German treatment guidelines. Psychotherapy based on trauma-focused cognitive behavioral therapy (Tf-CBT) is consistently recommended as the treatment of choice for PTSD in children and adolescents. Eye movement desensitization and reprocessing (EMDR) is also sometimes mentioned, but recommendations on this intervention are inconsistent. Only one guideline sees sufficient evidence to recommend EMDR as a first-line approach; the remaining guidelines advise that it should be considered only if Tf-CBT is unavailable or inapplicable. Additionally, there is a call for further research regarding this approach to improve the evaluation of its effectiveness. Psychopharmacology is consistently not recommended for children and adolescents, either as a stand-alone intervention or as an additional treatment option. For the treatment of complex PTSD, the considered guidelines emphasize the need for further research and consequently do not currently commit to a recommendation for a specific intervention. Existing trauma-focused treatments, however, are considered to be promising for not only successfully reducing PTSD symptom severity but also for use with cases of complex PTSD. However, before every treatment, it is imperative to conduct an age-appropriate collection of a differentiated trauma history in the form of a self-report and an external report using validated PTSD survey instruments. Moreover, the guidelines recommend that caregivers be closely involved in the therapy process. Although the number of controlled studies on the treatment of PTSD for children and adolescents has increased considerably in recent years - and the early and effective treatment of PTSD in children and adolescents can prevent the development of secondary disorders and clinically relevant suffering - guidelines in the adult field are still much more comprehensive and differentiated. Efforts should be made to increase the dissemination of empirically supported treatments by training key providers of children and adolescents such as licensed psychotherapists, psychotherapists in training, child and youth welfare agencies, and pediatricians. Future research should furthermore concentrate on evaluating EMDR for PTSD in children and adolescents and the treatment of complex PTSD in this age group.

Keywords: PTSD, trauma, children, adolescents, treatment, clinical guidelines

Über die Hälfte aller Kinder und Jugendlichen erleben vor der Vollendung ihres 18. Lebensjahres ein potentiell trau- matisches Ereignis (Landolt, Schnyder, Maier, Schoenbucher \& Mohler-Kuo, 2013). Entwickeln sich aus diesen 
Erlebnissen langfristige Stressreaktionen besteht das Risiko für eine Posttraumatischen Belastungsstörung (PTBS). Die PTBS wird nach der 10. Auflage der Internationalen statistischen Klassifikation der Krankheiten und verwandter Gesundheitsprobleme (ICD-10; World Health Organization, 1992) hauptsächlich durch die Symptombereiche Wiedererleben, Vermeidung und Überregung charakterisiert. Im forschungsorientierten diagnostischen und statistischen Leitfaden psychischer Störungen (DSM-5; American Psychiatric Association, 2013) beinhaltet die PTBS neben diesen drei Symptombereichen zusätzlich das Kriterium der negativen Veränderung von Kognition und Stimmung. Mit der ICD-11 (World Health Organization, 2018) wird es in Zukunft zusätzlich die neue Diagnose der komplexen PTBS geben. Die komplexe PTBS wird vergeben, wenn neben den klassischen PBTS-Symptomen Wiedererleben, Vermeidung und Überregung auch Störungen der Selbstorganisation wie interpersonelle Probleme, Emotionsregulationsschwierigkeiten, oder ein negatives Selbstkonzept festgestellt werden (World Health Organization, 2018). Bleiben diese Traumafolgestörungen unbehandelt, begleiten die gravierenden Langzeitfolgen die Betroffenen häufig bis ins Erwachsenenalter hinein und sind mit enormen Kosten für das Gesundheitssystem verbunden (Clemens et al. 2018; Ferrara et al., 2015; Thordardottir et al., 2016; Witt et al., 2019).

Viele in der Routineversorgung tätige Psychotherapeutinnen und Psychotherapeuten sind gegenüber evidenzbasierten Behandlungen kritisch eingestellt (Lilienfeld et al., 2013), was in der englischen Literatur unter dem Begriff "science-practitioner-gap“ adressiert wird. Im Bereich der PTBS Versorgung ist diese Lücke besonders groß: Hier existieren für alle Altersbereiche sehr gut empirisch evaluierte Behandlungsformen, aber viele in der stationären und ambulanten Routineversorgung tätige Psychotherapeutinnen und Psychotherapeuten wenden diese nicht an. Studien weisen auf eine mangelhafte Dissemination traumafokussierter Psychotherapien für Kinder und Jugendliche hin (Becker et al., 2004; Fegert et al., 2013; Ganser et al., 2016; Hipol \& Deacon, 2013). Einer der Gründe könnte in negativen Einstellungen der Behandlerinnen und Behandler bezüglich der Anwendung von expositionsbasierten Interventionen liegen (Farrell et al., 2013; Meyer et al., 2014; van Minnen et al., 2010) - es besteht die Sorge, dass eine traumafokussierte Psychotherapie zu einer Verschlimmerung der Symptomatik und dysfunktionalem Verhalten bei den Patientinnen und Patienten führen könnte - entsprechend zeigten Whiteside et al. (2016) im Rahmen einer Studie an 331 lizensierten Kinder- und Jugendlichenpsychotherapeutinnen und Therapeuten, dass Expositionselemente selten in der Behandlung eingesetzt wurden. Der Einsatz evidenzbasierter Verfahren in der Behandlung der PTBS bei Kin- dern und Jugendlichen ist wichtig, um langfristige chronische Störungsverläufe und die Ausbildung weiterer, komorbider Störungen durch eine möglichst frühe wirksame Behandlung der Traumafolgen zu verhindern. Klinische Leitlinien können den Behandlerinnen und Behandlern in der Routineversorgung hierbei Sicherheit vermitteln und den Einsatz effektiver Verfahren befördern.

Dieser Beitrag gibt einen Überblick über aktuelle Behandlungsempfehlungen klinischer Leitlinien im nationalen und internationalen Bereich und fasst die daraus resultierenden Implikationen für die Praxis und zukünftige Forschungsfragen zusammen.

\section{Internationale und nationale Leitlinien zur Behandlung der PTBS bei Kindern und Jugendlichen}

Klinische Leitlinien dienen unter anderem dem Zweck, Behandlerinnen und Behandlern sowie deren Patientinnen und Patienten die Auswahl geeigneter Diagnoseinstrumente und Therapien zu erleichtern (Hamblen et al., 2019). Von Expertengremien aus verschiedenen klinischen und wissenschaftlichen Bereichen werden Behandlungsempfehlungen mit differenziertem Stärkegrad ausgegeben. Bei der Festlegung des Stärkegrades ist zentral, dass die Grundlage für eine Therapieempfehlung eine möglichst aktuelle Evidenz darstellt, die transparent und verständlich kommuniziert wird (Institute of Medicine, 2011). Die Empfehlungen können dann bei der Auswahl einer Behandlung unterstützen, sind aber nicht als bindend anzusehen (Hamblen et al., 2019; Institute of Medicine, 2011).

Eine übersichtliche Zusammenfassung von Leitlinienempfehlungen im Bereich Traumatherapie (Forbes et al., 2010; Hamblen et al., 2019) sowie kritische Auseinandersetzungen mit der zugrundeliegenden Methodik (Courtois \& Brown, 2019; De Jongh et al., 2016; Silver \& Levant, 2019) oder den praktischen Implikationen (Henning \& Brand, 2019; Norcross \& Wampold, 2019) sind bisher hauptsächlich für erwachsene Patientinnen und Patienten vorhanden. Nach unserem Kenntnisstand gibt es nur eine Arbeit zu Kindern und Jugendlichen, die verschiedene Leitlinien auf den Einbezug interkultureller Kontexte überprüft (Alisic et al., 2020). Mit diesem Beitrag soll daher ein deutschsprachiger Überblick über aktuelle nationale und internationale Leitlinienempfehlungen zur Behandlung der PTBS gegeben werden, der sich explizit auf Patientinnen und Patienten im Kindes- und Jugendalter bezieht. 


\section{Vergleich der Leitlinienempfehlungen}

Um die Behandlungsempfehlungen darzustellen, werden internationale und die deutschen Leitlinien, die seit 2018 zur Behandlung der PTBS für Kinder und Jugendliche publiziert wurden, verglichen.

Bei den ausgewählten englischsprachigen Leitlinien handelt es sich um die international einflussreichsten und am meisten zitierten Leitlinien, die zudem eigene Datenanalysen vorgenommen haben. Diese stammen von drei internationalen Organisationen: dem National Institute for Health and Care Excellence (NICE, Großbritannien), der International Society for Traumatic Stress Studies (ISTSS) und dem Phoenix Australia Centre for Posttraumatic Mental Health (Phoenix Australia, Australien) in enger $\mathrm{Zu}-$ sammenarbeit mit dem National Health and Medical Research Council (NHMRC). Die deutschsprachige Leitlinie der Arbeitsgemeinschaft der Wissenschaftlichen Medizinischen Fachgesellschaften (AWMF, Deutschland) ist als neue nationale Leitlinie für Leser aus dem deutschsprachigen Raum von überragender Bedeutung.

In diesem Beitrag werden folgende Informationen bezüglich der einbezogenen klinischen Leitlinien beschrieben und verglichen: zum einen werden die Herausgeber, das Erscheinungsjahr und die Zusammensetzung der Expertengremien vorgestellt. Zum anderen werden die Evidenzgrundlagen der Leitlinien sowie deren Bewertungen und daraus resultierende Stärkegrade der Behandlungsempfehlungen erörtert. Für jede Leitlinie wird außerdem dargestellt, was die Methode erster Wahl zur Behandlung der PTBS bei Kindern und Jugendlichen ist. Außerdem werden weitere verfügbare Therapieoptionen sowie der zugehörige Empfehlungsgrad präsentiert. Falls vorhanden, wird zusätzlich auf den Inhalt und Hinweise zur Durchführung der Behandlung eingegangen. Außerdem wird verglichen, welche Empfehlungen die Leitlinien zur Behandlung von Kindern und Jugendlichen hinsichtlich Pharmakotherapie und komplexer PBTS geben.

Eine Übersicht über die einbezogenen klinischen Leitlinien sowie eine Zusammenfassung der wichtigsten Ergebnisse des Vergleiches kann Tabelle 1 entnommen werden.

\section{AWMF-Leitlinien}

In Deutschland wurde 2019 die zweite Auflage der S-3 Leitlinien Posttraumatische Belastungsstörung (Schäfer et al., 2019) der AWMF herausgegeben. Die AWMF ist ein Zusammenschluss diverser medizinischer und psychologischer Fachgesellschaften, die in verschiedenen gesundheitlichen Bereichen beratend tätig ist. Als Datengrundlage für die S3-Leitlinien zur PTBS dienten bereits veröf- fentlichte nationale und internationale Leitlinien bis zum Jahr 2014, systematische Reviews sowie alleinstehende randomisierte kontrollierte Studien (randomized controlled trials; RCTs). Bezüglich des Empfehlungsgrades sind vier verschiedene Stufen vorgesehen, die sich an der Qualität der zugrundeliegenden Evidenz orientieren: starke Empfehlung (Empfehlungsgrad A), mittlere Empfehlung (Empfehlungsgrad B), keine Empfehlung (Empfehlungsgrad 0) sowie der klinische Konsenspunkt (KPP). Die letzte Kategorie bezieht sich auf den Konsens klinischer Expertinnen und Experten und basiert somit nicht auf einer Evidenzbewertung (Schäfer et al., 2019).

Als Behandlung erster Wahl soll eine Traumafokussierte Kognitive Verhaltenstherapie (Tf-KVT) jedem Kind oder Jugendlichen mit PTBS angeboten werden (Empfehlungsgrad A). Den höchsten Evidenzgrad sprechen die AWMF-Leitlinien der Tf-KVT nach dem Manual von Cohen, Mannarino \& Deblinger (2009) zu. Eye Movement Desensitization and Reprocessing (EMDR; Shapiro, Wesselmann \& Mevissen, 2017) wird als Psychotherapieform für Kinder und Jugendliche mit PTBS als vielversprechend, aber nicht genügend abgesichert eingeschätzt. Gleiches gilt für die Narrative Expositionstherapie für Kinder (KidNET; Schauer, Neuner \& Elbert, 2017).

Eine Pharmakotherapie soll hingegen nicht in der Therapie der PTBS bei Kindern und Jugendlichen eingesetzt werden. Insbesondere sollen aufgrund ihres Suchtpotenzials keine Benzodiazepine eingesetzt werden (Empfehlungsgrad A). Darüber hinaus wird empfohlen, die Eltern oder eine andere Bezugsperson in den Therapieprozess einzubinden (Empfehlungsgrad B). Auf Basis klinischer Konsenspunkte (KPP) werden weitere Hinweise (Empfehlungsgrad KPP) zur Durchführung der Behandlung gegeben. So soll die Therapie an den individuellen Entwicklungsstand angepasst und Komorbiditäten bei der Therapieplanung berücksichtigt werden. Darüber hinaus ist eine Anmerkung der Leitlinien, dass neben der traumafokussierten Intervention zusätzliche Symptombereiche, wie beispielsweise Aggressivität oder der Selbstwert von Betroffenen, in der Therapie thematisiert werden sollten. Vor einer Intervention wird jedoch nahegelegt, dass die Sicherstellung des Kindeswohles bei potentieller Gefährdung an erster Stelle steht (Empfehlungsgrad KPP; Rosner et al., 2019).

Weitere Klinische Konsenspunkte betreffen die Empfehlungen zur Behandlung der Komplexen PTBS (laut ICD-11 Vorschlag definiert). Hier sollte die psychotherapeutische Behandlung mit einer Kombination traumafokussierter Techniken erfolgen, bei welcher Schwerpunkte auf der Verarbeitung von Erinnerungen an die traumatischen Erlebnisse und/oder ihrer Bedeutung liegen sowie auf Techniken zur Emotionsregulation und zur Verbesserung von Bindungsproblemen. Ergänzend zu traumafo- 
Tabelle 1. Klinische Behandlungsleitlinien der PTBS für Kinder und Jugendliche

\begin{tabular}{|c|c|c|c|c|c|c|c|}
\hline Jahr & $\begin{array}{l}\text { Evidenz- } \\
\text { grundlage }\end{array}$ & Bewertung der Evidenz & $\begin{array}{l}\text { Stärke der } \\
\text { Empfehlung }\end{array}$ & Altersstufen & $\begin{array}{l}\text { Behandlung } \\
\text { erster Wahl }\end{array}$ & $\begin{array}{l}\text { Empfehlung } \\
\text { für Pharma- } \\
\text { kotherapie }\end{array}$ & $\begin{array}{l}\text { Informationen } \\
\text { zu KPTBS }\end{array}$ \\
\hline AWMF 2019 & $\begin{array}{l}\text { Andere Leitlinien } \\
\text { bis } 2014 \\
\text { Vorhandene Sys- } \\
\text { tematische Re- } \\
\text { views, } \\
\text { Alleinstehende } \\
\text { RCTs }\end{array}$ & $\begin{array}{l}\text { Systematische Übersichts- } \\
\text { arbeiten hochwertiger } \\
\text { RCTs (la), einzelne RCTs } \\
\text { (Ib), einzelne kontrollierte } \\
\text { Studien ohne Randomisie- } \\
\text { rung (II), Korrelations-, Ver- } \\
\text { gleichsstudien, Fallberich- } \\
\text { te (III) }\end{array}$ & $\begin{array}{l}\text { starke Empfeh- } \\
\text { lung (A), mittlere } \\
\text { Empfehlung (B), } \\
\text { keine Empfeh- } \\
\text { lung (0), klini- } \\
\text { scher Konsens- } \\
\text { punkt (KPP) }\end{array}$ & $\begin{array}{l}\text { Kinder (0 - } \\
14 \text { Jahre), Ju- } \\
\text { gendliche (14- } \\
18 \text { Jahre) }\end{array}$ & Tf-KVT & Nein & Ja \\
\hline NICE 2018 & $\begin{array}{l}\text { Teilaktualisierung } \\
\text { der Leitlinien aus } \\
2005, \\
\text { vorhandene Sys- } \\
\text { tematische Re- } \\
\text { views, } \\
\text { alleinstehende } \\
\text { RCTs }\end{array}$ & $\begin{array}{l}\text { Hoch (unwahrscheinlich, } \\
\text { dass weitere Untersuchun- } \\
\text { gen Einschätzung ändern), } \\
\text { moderat (wahrscheinlich, } \\
\text { dass weitere Untersuchun- } \\
\text { gen Einschätzung ändern), } \\
\text { niedrig (sehr wahrschein- } \\
\text { lich, dass weitere Untersu- } \\
\text { chungen Einschätzung än- } \\
\text { dern), sehr niedrig (die Ein- } \\
\text { schätzung der Wirksamkeit } \\
\text { ist sehr unsicher) }\end{array}$ & $\begin{array}{l}\text { Sollte angeboten } \\
\text { werden (anbie- } \\
\text { ten/ nicht anbie- } \\
\text { ten), könnte an- } \\
\text { geboten werden } \\
\text { (in Betracht zie- } \\
\text { hen/nicht in Be- } \\
\text { tracht ziehen) }\end{array}$ & $\begin{array}{l}\text { Kinder \& junge } \\
\text { Erwachsene (< } \\
18 \text { Jahre) }\end{array}$ & Tf-KVT & Nein & Ja \\
\hline ISTSS 2019 & $\begin{array}{l}\text { Eigens erstellte } \\
\text { systematische } \\
\text { Reviews, allein- } \\
\text { stehende RCTs }\end{array}$ & $\begin{array}{l}\text { Hoch (unwahrscheinlich, } \\
\text { dass weitere Untersuchun- } \\
\text { gen Einschätzung ändern), } \\
\text { moderat (wahrscheinlich, } \\
\text { dass weitere Untersuchun- } \\
\text { gen Einschätzung ändern), } \\
\text { niedrig (sehr wahrschein- } \\
\text { lich, dass weitere Untersu- } \\
\text { chungen Einschätzung än- } \\
\text { dern), sehr niedrig (die Ein- } \\
\text { schätzung der Wirksamkeit } \\
\text { ist sehr unsicher) }\end{array}$ & $\begin{array}{l}\text { Starke Empfeh- } \\
\text { lung (dafür/da- } \\
\text { gegen), mittlere } \\
\text { Empfehlung (da- } \\
\text { für/dagegen), } \\
\text { Intervention mit } \\
\text { niedriger Evi- } \\
\text { denz, unzurei- } \\
\text { chende Evidenz } \\
\text { für eine Empfeh- } \\
\text { lung, Interventi- } \\
\text { on mit aufkom- } \\
\text { mender Evidenz }\end{array}$ & $\begin{array}{l}\text { Kinder \& Ju- } \\
\text { gendliche (bis } \\
18 \text { Jahre) }\end{array}$ & $\begin{array}{l}\text { Tf-KVT, } \\
\text { EMDR }\end{array}$ & Nein & $\mathrm{Ja}$ \\
\hline $\begin{array}{l}\text { Phoenix } \\
\text { Australia } 2020\end{array}$ & $\begin{array}{l}\text { Reviews der } \\
\text { ISTSS, Update } \\
\text { durch aktuellere } \\
\text { RCTs }\end{array}$ & $\begin{array}{l}\text { Die Evidenz kann als Leit- } \\
\text { faden für die Praxis dienen } \\
\text { (A), Die Evidenz kann in den } \\
\text { meisten Situationen als } \\
\text { Leitfaden für die Praxis } \\
\text { dienen (B), Die Evidenz bie- } \\
\text { tet eine gewisse Unterstüt- } \\
\text { zung für die Empfehlungen, } \\
\text { Anwendung aber mit Vor- } \\
\text { behalt (C), } \\
\text { Evidenz ist schwach, Emp- } \\
\text { fehlungen müssen mit Vor- } \\
\text { sicht angewendet werden } \\
\text { (D) }\end{array}$ & $\begin{array}{l}\text { Starke Empfeh- } \\
\text { lung (dafür/da- } \\
\text { gegen), mittlere } \\
\text { Empfehlung (da- } \\
\text { für/dagegen) }\end{array}$ & $\begin{array}{l}\text { Vorschulkinder } \\
\text { ( } 0 \text { bis } 8 \text { Jahre), } \\
\text { Schulkinder } \\
\text { ( } 9-11 \text { Jahre), } \\
\text { Jugendliche } \\
\text { (12-17 Jahre) }\end{array}$ & Tf-KVT & $\begin{array}{l}\text { Keine Anga- } \\
\text { be }\end{array}$ & Ja \\
\hline
\end{tabular}

Anmerkungen: AWMF = Arbeitsgemeinschaft der Wissenschaftlichen Medizinischen Fachgesellschaften; NICE = Nationale Institute for Health and Care Excellence; ISTSS = International Society for Traumatic Stress Studies; Phoenix Australia = Phoenix Australia Centre for Posttraumatic Mental Health; RCTs = randomized controlled trials [randomisierte kontrollierte Studien]; Tf-KVT = Traumafokussierte kognitive Verhaltenstherapie; EMDR = Eye Movement Desensitization and Reprocessing.

kussierten Interventionen sollen weitere Probleme und Symptombereiche abgeklärt und in der Behandlung berücksichtigt werden, wie z.B. das Risiko erneuter Viktimisierung bei Opfern von Gewalt, Aggressivität, Trauer- prozesse, soziale Neuorientierung, Neubewertung und Selbstwertstabilisierung. Bei schwerwiegenden komorbiden Störungen bzw. Symptomen und akuter Suizidalität können vor dem Einsatz traumafokussierter Interventio- 
nen geeignete Interventionen zur Therapie dieser Störungen durchgeführt werden. Zu Beginn der Behandlung sollte die aktuelle Gefährdung des Kindes bzw. des Jugendlichen (z. B. anhaltende Bedrohung durch Täter) abgeklärt werden. Bei anhaltender Bedrohung sollen geeignete Maßnahmen zur Sicherung des Kindeswohles vorrangig ergriffen werden (Empfehlungsgrad KPP; Rosner et al., 2019).

\section{NICE-Leitlinien}

Mit dem National Institute for Health and Care Excellence (NICE) existiert in Großbritannien eine öffentliche Einrichtung, welche Leitlinien für die staatliche nationale Gesundheitsversorgung sowie Bereiche der Sozialfürsorge erstellt. Die NICE Leitlinie NG116 Postraumatic Stress Disorder wurde von PTBS Expertinnen und Experten aus der klinischen Praxis und Forschung entwickelt (National Institute for Health and Care Excellence, 2018). Das NICE führte mit der Erstellung dieser Leitlinie eine Teilaktualisierung der vorherigen Leitlinien aus dem Jahr 2005 durch, wobei die dort beschriebenen Erkenntnisse übernommen und bei Bedarf auf der Grundlage aktueller Daten erneuert wurden. Die Evidenzgrundlage der aktuellen Leitlinien bilden systematische Reviews sowie einzelne RCTs bis zum Januar 2018. Die Stärke der ausgesprochenen Empfehlungen ist zweistufig aufgebaut. Es wird unterschieden zwischen Behandlungen, die angeboten werden sollten (starke Empfehlung) und Behandlungen, die angeboten werden könnten (mittlere Empfehlung). Im zweiten Fall sollte eine Anwendung lediglich in Betracht gezogen werden (National Institute for Health and Care Excellence, 2018).

Auch die NICE Leitlinien beschreiben die individuell administrierte Tf-KVT als Methode erster Wahl für die Behandlung von Kindern und Jugendlichen mit PTBS oder klinisch bedeutsamen Symptomen nach einer Traumatisierung (starke Empfehlung). Dies trifft für Patientinnen und Patienten im Alter von sieben bis 17 Jahren zu, bei denen die Symptomatik drei Monate nach dem traumatischen Ereignis weiterhin Bestand hat. Vor Ablauf dieser drei Monate bzw. bei Kindern im Alter von 5 bis 6 Jahren kann eine individuelle Tf-KVT ebenfalls in Betracht gezogen werden (mittlere Empfehlung). Die Leitlinie macht darüber hinaus präzise Angaben zur konkreten Durchführung der Tf-KVT. So sollte ein validiertes Manual genutzt, sechs bis 12 Sitzungen durchgeführt (mehr wenn das Kind Opfer multipler Traumatisierung wurde), und die Behandlung durch trainierte Klinikerinnen oder Kliniker unter Supervision durchgeführt werden. Die Behandlung sollte an Alter und Entwicklungsstand des Kindes angepasst sein sowie Eltern bzw. Bezugspersonen einschließen. Sie sollte die Elemente Psychoedukation über die Reaktionen auf Traumata, Strategien zur Emotionsregulation und zum Umgang mit Flashbacks sowie Pläne zur Herstellung einer sicheren Umgebung für das Kind beinhalten. Darüber hinaus sollte die Elaboration und Bearbeitung traumatischer Erinnerungen, die Bearbeitung traumabezogener Emotionen wie Scham, Schuld, Trauer und Ärger, die Restrukturierung der Bedeutung des Traumas, sowie Hilfe bei der Überwindung von Vermeidung im Fokus der Behandlung stehen. Wenn nötig, sollten Boostersitzungen geplant werden, vor allem in Verbindung mit wichtigen Ereignissen wie Jahrestagen (National Institute for Health and Care Excellence, 2018).

Eine Behandlung mit EMDR kann für Kinder und Jugendliche zwischen sieben und 17 Jahren mit PTBS oder klinisch bedeutsamen Symptomen nach einer Traumatisierung dann in Erwägung gezogen werden, wenn diese auf die Tf-KVT nicht ansprechen oder sich nicht auf die Tf-KVT einlassen können.

Von Pharmakotherapie für Kinder und Jugendliche mit PBTS wird abgeraten (starke Empfehlung). Die Leitlinien bieten außerdem Anhaltspunkte für individuelle sowie gruppenbasierte Interventionsprogramme zur Prävention der PBTS im Kindes- und Jugendalter. Bei Fällen mit komplexer PTBS werden zwar die Besonderheiten in der Psychopathologie und der Diagnosestellung beschrieben, aber im Gegensatz zu den deutschen Leitlinien keine gesonderten Aussagen zu deren Behandlung getroffen (National Institute for Health and Care Excellence, 2018).

\section{ISTSS-Leitlinien}

Nicht nur auf nationaler Ebene innerhalb Europas, sondern auch im internationalen Kontext werden Empfehlungen für psychotherapeutische Behandlungen der PTBS bei Kindern und Jugendlichen formuliert. Die ISTSS ist eine weltweit tätige Gesellschaft, die die Weiterentwicklung und den Austausch von Wissen und Erkenntnissen über die Auswirkungen traumatischer Ereignisse fördert. Im Jahr 2019 erschien die Zusammenfassung zur dritten Überarbeitung der PTSD prevention and treatment guidelines methodology and recommendations (ISTSS Guidelines Committee, 2019). Das zugehörige Buch, welches die Hauptkomponente der mehrgliedrigen Leitlinien darstellt, folgte ein Jahr später (Forbes, Bisson, Monson \& Berliner, 2020). Das Entwicklerkomitee, bestehend aus internationalen Expertinnen und Experten der PTBS mit wissenschaftlichem Hintergrund, spricht Behandlungsempfehlungen auf der Grundlage eigens erstellter systematischer Reviews aus. Bereits bestehende Reviews wurden um alleinstehende RCTs aus dem Zeitraum von Januar bis März 2018 ergänzt. Starke Empfehlungen basie- 
Tabelle 2. Beschreibung der Tf-KVT und EMDR zur Behandlung der PTBS bei Kindern und Jugendlichen

\begin{tabular}{ll}
\hline Intervention & Hauptmerkmale \\
\hline Tf-KVT & Basis: Kognitive Verhaltenstherapie \\
& Traumafokussierte Techniken zentral (imaginative Exposition, narrative Exposition, Exposition in vivo, kognitive Umstrukturierung \\
& von Überzeugungen mit Bezug zum Trauma) \\
& Verschiedene Ansätze und Manuale innerhalb der Tf-KVT unterscheiden sich bezüglich Anzahl der Therapieeinheiten und Dosie- \\
& rung, Anteil von kognitiven im Verhältnis zu expositionsbasierten Elementen, Ausmaß des Einbezugs der Bezugspersonen in die \\
& Behandlung und dem angenommenen Wirkmechanismus \\
& Manual mit größter Evidenz: Tf-KVT nach Cohen et al. (2009) \\
& Traumafokussierte Intervention mit acht Phasen \\
& Ziel ist die weitreichende Erneuerung des pathogenen Erlebnisses \\
& Eingesetzte Elemente sind bilaterale Stimulation durch Augenbewegungen, Klopfen oder Töne sowie kurze Expositionsphasen \\
& Ausgewählte traumatische Erinnerungen werden in Form von vier zusammenhängenden Komponenten betrachtet: visuelles Bild, \\
& Kognition, Affekt, Körperempfindung \\
& Manual zu EMDR im Kindes- und Jugendalter: Shapiro et al. (2017)
\end{tabular}

ren auf Interventionen mit qualitativ hochwertiger Evidenz und einer hohen Sicherheit für deren Wirksamkeit. Eine Standardempfehlung gilt für Behandlungsansätze, deren Wirksamkeit mit einer weniger großen Sicherheit nachgewiesen werden kann. Drei weitere Stärkegrade beziehen sich auf Interventionen, für die weder eine starke noch eine Standardempfehlung festgelegt wird: Interventionen mit niedriger Evidenz, Interventionen mit zunehmender Evidenz und Interventionen mit unzureichender Evidenz für eine Empfehlung (Bisson et al., 2019).

Als Therapie erster Wahl für Kinder und Jugendliche werden von der ISTSS zwei verschiedene Behandlungsansätze (jeweils starke Empfehlung) genannt: die Tf-KVT (Kind/Jugendlicher alleine oder gemeinsam mit einer Bezugsperson) und EMDR werden bei klinisch relevanter PTBS-Symptomatik empfohlen. Die Hauptmerkmale dieser beiden Behandlungsmethoden können Tabelle 2 entnommen werden.

Darüber hinaus beschreiben die Autorinnen und Autoren eine zunehmende Evidenz für die Wirksamkeit einer Behandlung mit gruppenbasierter Tf-KVT, Psychoedukationsgruppen und Interventionen zur Verbesserung der Eltern-Kind-Beziehung. Folgende psychotherapeutischen Ansätze haben nach den Leitlinien unzureichende Evidenz für eine Empfehlung: Tf-KVT, wenn sie ausschließlich mit einer Bezugsperson durchgeführt wird, Familientherapie, gruppenbasierte Tf-KVT gemeinsam mit Kindern und Bezugspersonen, KidNET, unspezifische Beratung sowie Stepped-Care Tf-KVT (gemeinsam mit Bezugsperson und Kind). Bei der Stepped-Care Tf-KVT handelt es sich um eine Intervention, bei welcher vor der therapeutengeleiteten Tf-KVT Therapie eine von den Eltern geleitete Behandlung durchgeführt wird. Der Therapeut oder die Therapeutin und webbasierte Materialien unterstützen die Eltern bei der Durchführung. Wenn nach dieser Intervention dann noch ein Behandlungsbedarf besteht, er- halten die Kinder oder Jugendlichen die vollständige, therapeutengeleitete Tf-KVT.

Im Bereich der Pharmakotherapie ist nach den ISTSSLeitlinien ebenfalls unzureichende Evidenz für die Empfehlung einer Behandlung von Kindern und Jugendlichen mit Sertralin vorhanden (ISTSS Guidelines Committee, 2019).

In einem Positionspapier zur komplexen PTBS wird festgehalten, dass es derzeit noch keine ausreichende Evidenzgrundlage gibt, um eine spezifische Intervention für die Behandlung der komplexen PTBS bei Kindern zu empfehlen. Allerdings enthalten viele traumafokussierte Therapien Komponenten, die sowohl die PTBS Symptome als auch die Symptome der komplexen PTBS (negatives Selbstkonzept, Emotionsregulationsprobleme, Beziehungsschwierigkeiten) adressieren und in beiden Bereichen Behandlungserfolge erzielen. (ISTSS Guidelines Committee, n.d.).

\section{Phoenix Australia-Leitlinien}

Phoenix Australia orientierte sich bei der Erstellung der australischen Leitlinien im Wesentlichen an den systematischen Reviews der ISTSS. Phoenix Australia ist eine gemeinnützige Organisation (früher bekannt als Australian Centre for Posttraumatic Mental Health), welche in enger Abstimmung mit dem National Health and Medical Research Council (NHMRC) in 2020 die dritte Überarbeitung der Australian Guidelines for the Prevention and Treatment of Acute Stress Disorder, Posttraumatic Stress Disorder and Complex PTSD publizierte (Phoenix Australia Centre for Posttraumatic Mental Health, 2020). Das Expertengremium der Entwicklergruppe bestand aus führenden australischen PTBS-Expertinnen und Experten aus der Wissenschaft sowie klinischen Versorgenden, welche mit traumatisierten Patientinnen und Patienten arbeiten. Die ISTSS Reviews wurden um zusätzliche, 
neuere Befunde aus dem Zeitraum von Oktober 2018 bis Juni 2019 ergänzt. Die Stärke der Empfehlungen variiert in diesen Leitlinien zwischen einer starken Empfehlung oder einer bedingten Empfehlung (Phoenix Australia Centre for Posttraumatic Mental Health, 2020).

Auch diese Leitlinien sprechen eine starke Empfehlung für die Anwendung der Tf-KVT bei Kindern und Jugendlichen mit klinisch relevanten PTBS-Symptomen aus. Dabei kann die Tf-KVT sowohl mit dem Kind alleine als auch gemeinsam mit einer Bezugsperson durchgeführt werden. Der Empfehlungsgrad für diese beiden Optionen (starke Empfehlung) unterscheidet sich nicht. Wenn möglich, sollen Eltern oder Bezugspersonen jedoch während der gesamten Behandlung mit einbezogen werden, um das Kind oder den Jugendlichen zu unterstützen und eine positive Erziehung zu fördern. Falls die Tf-KVT nicht zugänglich oder für das Kind nicht akzeptabel ist, wird empfohlen, Kindern und Jugendlichen mit PTBS Symptomen EMDR anzubieten (bedingte Empfehlung). Für die Anwendung bei Kindern werden Modifikationen des EMDR-Protokolls entsprechend des Alters und des Entwicklungsstandes vorgenommen.

Zur Pharmakotherapie bei PTBS werden in dieser Leitlinie keine Angaben zu Kindern und Jugendlichen gemacht und es wird ausschließlich das Vorgehen bei erwachsenen Betroffenen beschrieben. In gesonderten Kapiteln finden sich außerdem detaillierte Ausführungen zu Besonderheiten der PTBS bei spezifischen Populationen wie beispielsweise Geflüchteten oder Opfern von sexuellen Übergriffen. Kindern und Jugendlichen mit PTBS, die sexuell misshandelt wurden, sollte beispielsweise ebenfalls eine Tf-KVT angeboten werden, die für ihr Alter und ihren Entwicklungsstand angemessen ist. Die Leitlinien legen darüber hinaus nahe, dass Fachkräfte oft zu komplexe Ausdrücke verwenden, wenn sie mit Kindern über sexuelle Übergriffe sprechen. Es sei wichtig, abstrakte und komplizierte Fragen zu vermeiden und sich mit möglichsten einfachen Worten konkret auszudrücken. Darüber hinaus kann es hilfreich sein, in der Therapie eine Auswahl sicherer Verhaltensweisen zu besprechen, um das Gefühl der Kontrolle über die Umgebung bei Betroffenen zu stärken. Dies ist besonders wichtig, wenn sexuelle Misshandlung im Umfeld eines desorganisierten Familienlebens auftritt (Phoenix Australia Centre for Posttraumatic Mental Health, 2020).

Im Kapitel zur komplexen PTBS wird dargelegt, dass es aktuell noch keine direkte Evidenz dafür gibt, wie Kindern und Jugendliche mit komplexer PTBS behandelt werden sollten. Es wird darauf hingewiesen, dass in der Literatur diskutiert wird, ob die derzeitigen evidenzbasierten Behandlungen für PTBS bei Menschen mit komplexer PTBS wirksam sind oder modifiziert werden müssen (Phoenix Australia Centre for Posttraumatic Mental Health, 2020).

\section{Fazit zum Vergleich der Leitlinienempfehlungen}

Übereinstimmend empfehlen alle oben aufgeführten klinischen Leitlinien eine traumafokussierte Psychotherapie zur Behandlung der PTBS für Kinder und Jugendliche. Die Methode erster Wahl ist die Tf-KVT, die mit dem Kind oder Jugendlichen alleine oder gemeinsam mit einer Bezugsperson durchgeführt werden kann (ISTSS Guidelines Committee, 2019; National Institute for Health and Care Excellence, 2018; Phoenix Australia Centre for Posttraumatic Mental Health, 2020; Rosner et al., 2019). Bezüglich des Einsatzes von EMDR herrscht Uneinigkeit. In den deutschen Leitlinien wird eine Forderung nach weiterer Forschung auf diesem Gebiet gestellt und folglich keine Empfehlung für eine Behandlung mit EMDR ausgesprochen (Rosner et al., 2019). Sowohl das NICE als auch Phoenix Australia empfehlen EMDR als Behandlung zu erwägen, falls die TF-KVT nicht zugänglich, nicht akzeptabel oder unwirksam ist (National Institute for Health and Care Excellence, 2018; Phoenix Australia Centre for Posttraumatic Mental Health, 2020). Die ISTSS Leitlinien sprechen dagegen eine starke Empfehlung für EMDR als Methode erster Wahl aus und stellen diese somit auf die gleiche Evidenzstufe wie die Tf-KVT (ISTSS Guidelines Committee, 2019), trotz der unterschiedlich breiten empirischen Datenlage für diese beiden Verfahren.

Einheitlicher sind die Stellungnahmen bezüglich medikamentöser Therapien der PTBS. Zur Psychopharmakologie wird für Kinder und Jugendliche übereinstimmend weder als alleinstehende Intervention noch als zusätzliche Behandlungsoption geraten (National Institute for Health and Care Excellence, 2018; ISTSS Guidelines Committee, 2019; Rosner et al., 2019).

Zur Behandlung der komplexen PTBS unterstreichen vier Leitlinien die Notwendigkeit weiteren Forschungsbedarfs und legen sich folglich aktuell noch nicht auf eine Empfehlung für eine spezifische Intervention fest (ISTSS Guidelines Committee, 2019; Phoenix Australia Centre for Posttraumatic Mental Health, 2020; Rosner et al., 2019).

Obwohl die Anzahl kontrollierter Studien zur Behandlung der PTBS für Kinder und Jugendliche in den vergangenen Jahren stark zugenommen hat, und eine frühe und wirksame Behandlung der PTBS bei Kindern und Jugendlichen die Ausbildung sekundärer Störungsbilder sowie klinisch relevantes Leid verhindern kann, sind Leitlinien im Erwachsenenbereich immer noch deutlich umfassender und differenzierter. Die aktuellen Leitlinien der American Psychological Association (APA) und des Department of Veterans Affairs and the Department of Defense (VA/DoD) beziehen sich beispielsweise ausschließlich auf die Behandlung von volljährigen Patientinnen und Pati- 
enten und machen keine Angaben zu Kindern oder Jugendlichen (American Psychological Association, 2017; Department of Veterans Affairs and Department of Defense, 2017).

Bei der Interpretation der klinischen Leitlinien sollte grundsätzlich beachtet werden, dass diese auf verschiedenen methodischen Ansätzen beruhen und sich daher auch auf unterschiedliche Evidenzgrundlagen beziehen. Schon leichte Unterschiede in der Suchstrategie für die zugrundeliegende Literatur oder der methodischen Fragestellungen können hinterher große Auswirkungen auf die gegebenen Empfehlungen haben (Hamblen et al., 2019). Zwar beziehen sich alle beschriebenen Leitlinien auf die Ergebnisse von RCTs, dennoch variieren die Einschlusskriterien für diese Studien. Beispielsweise berücksichtigt die ISTSS ausschließlich individuelle RCTs und eigene Übersichtsarbeiten, wohingegen beim NICE bei ausgewählten Fragestellungen auch bereits vorhandene Reviews oder qualitative Studien eingeflossen sind. Auch die Kriterien für Studienpopulationen, Outcome-Kriterien und diagnostische Grundlagen werden unterschiedlich präzise definiert. Die ISTSS gibt beispielsweise als alleinige Leitlinie vor, dass die PTBS-Diagnose der Probandinnen und Probanden in RCTs auf der Basis strukturierter oder klinischer Interviews gestellt worden sein muss. Zusätzlich sollte auf die Variationen bei der Graduierung der Empfehlungen geachtet werden, da jede klinische Leitlinie ihre eigenen Kategorien festlegt und unterschiedliche Kriterien zur Evidenzbewertung zugrunde liegen (vgl. Tabelle 1).

An dieser Stelle sollten außerdem auch allgemeinere Kritikpunkte an klinischen Leitlinien benannt und diskutiert werden. Evidenzbasierte Leitlinien beruhen, wie bereits häufig erwähnt, auf RCTs zur Wirksamkeit von Interventionen. So werden beispielsweiße in vielen Studien zur Behandlung der PTBS junge Patientinnen und Patienten mit schweren komorbiden Störungen oder Symptomen, wie z.B. schwerem selbstverletzendem Verhalten oder häufiger suizidaler Ideation, ausgeschlossen. Komorbid vorliegende, weitere psychische Störungen und Probleme sind aber eher die Regel als die Ausnahme bei der PTBS des Kindes- und Jugendalters, besonders infolge der Erfahrung von physischer oder sexualisierte Gewalt (Chen et al., 2010). Ferner wird oft kritisiert, dass die verschiedenen Therapieschulen ein sich unterscheidendes Wissenschaftsverständnis haben. Kognitiv-behaviorale Interventionen werden häufiger mit Hilfe von RCTs untersucht als tiefenpsychologische oder psychoanalytische Interventionen. So bleibt offen, ob diese Interventionen wirksam sind oder nicht, weil sie zu wenig untersucht werden (Courtois \& Brown, 2019). Für die Behandlung von Vorschulkindern mit PTBS liegen insgesamt vergleichsweisen wenige Evidenzen vor - hier geben da- her auch Leitlinien vergleichsweise wenig Orientierung (vgl. Stauffer et al., 2020). Weiterhin wurde von Alisic et al. (2020) bemängelt, dass der kulturelle Hintergrund von Patientinnen und Patienten und ihren Familien wenig berücksichtigt wird. Allerdings lassen andere Übersichten und Einzelstudien auch die Vermutung zu, dass manche Therapiemanuale interkulturell sensibel genug sind um von der individuellen Therapeutin oder dem Therapeuten angepasst zu werden (Nocon et al, 2017, Unterhitzenberger et al, 2019). Vergleiche von kulturell angepassten und kulturell sensiblen Interventionen in RCT Designs fehlen. Des Weiteren werden Leitlinien auch deswegen kritisiert, weil sie die Meinung und Wünsche von Patientinnen und Patienten im Entstehungsprozess nicht einbeziehen (vgl. Alisic et al., 2020).

\section{Implikationen und Ausblick}

Bestehende nationale und internationale Leitlinien empfehlen übereinstimmend zur Behandlung der PTBS bei Kindern und Jugendlichen Psychotherapie in Form von Tf-KVT und, zum Teil, EMDR. Dennoch mangelt es an der Dissemination von empirisch unterstützten Psychotherapien in der Routineversorgung (Ganser, Münzer, Plener, Witt \& Goldbeck, 2016; Münzer, Fegert, \& Goldbeck, 2015, Rosner et al., 2020). Um diese voranzutreiben, ist ein Training der Psychiaterinnen und Psychiater sowie Psychotherapeutinnen und Psychotherapeuten notwendig, die im stationären, teilstationären und ambulanten Rahmen die Versorgung der betroffenen jungen Menschen sicherstellen (Chorpita \& Daleiden, 2014; Sholomskas et al., 2005). Dieses Training sollte auch bereits in die Ausbildung der beschriebenen Berufsgruppen aufgenommen werden. Eine kostenfreie und unkompliziert $\mathrm{zu}$ nutzende Fortbildungsmöglichkeit bietet das „Online-Training für Therapeuten in der traumafokussierten kognitiven Verhaltenstherapie für Kinder und Jugendliche" (https://tfkvt.ku.de) der Katholischen Universität Eichstätt unter der Leitung von Prof. Dr. Rita Rosner.

Kinderärztinnen und Kinderärzte sowie Mitarbeitende der Jugendhilfe sollten über die bestehenden Behandlungsoptionen informiert sein, um so betroffene Kindern und Jugendliche unterstützen zu können, damit diese möglichst umgehend nach einer PTBS Diagnose die optimale Hilfe erhalten (Rosner et al., 2020). Die Aufgaben für die zukünftige Psychotherapieforschung liegen in der Durchführung weiterer Studien zur Wirksamkeit von EMDR bei PTBS im Kindes- und Jugendalter und in der Klärung der Frage, ob die komplexe PTBS bei Kindern und Jugendlichen mit den derzeit vorliegenden evidenzbasierten Psychotherapien behandelt werden kann. $\mathrm{Zu}-$ 
sätzlich sollte evaluiert werden, ob ein Vorgehen abweichend von den bisher wirksamen Interventionsformen, in der Behandlung komplexer Traumatisierung bei jungen Menschen größere Erfolge erzielen kann. Spezifisch bedeutet dies, dass in RCTs untersucht werden sollte, ob die in den Leitlinien für die PTBS empfohlenen Interventionen mit neuen Techniken erweitert werden müssen (z. B. hinsichtlich einem intensiveren Fokus auf Emotionsregulation) oder ob nur die Therapiedosis und damit die Dauer erhöht werden sollte.

\section{Literatur}

Alisic, E., Roth, J., Cobham, V., Conroy, R., De Young, A., Hafstad, G. et al. (2020). Working towards inclusive and equitable trauma treatment guidelines: a child-centered reflection. European Journal of Psychotraumatology, 11, 1833657. https://doi.org/ 10.1080/20008198.2020.1833657

American Psychological Association (APA). (2017). Clinical practice guideline for the treatment of posttraumatic stress disorder (PTSD) in adults. Washington, DC: APA. https://www.apa.org/ ptsd-guideline/ptsd.pdf

American Psychiatric Association (APA). (2013). Diagnostic and statistical manual of mental disorders: fifth edition (DSM-5). Washington, DC: APA.

Bisson, J. I., Berliner, L., Cloitre, M., Forbes, D., Jensen, T. K., Lewis, C. et al. (2019). The international society for traumatic stress studies new guidelines for the prevention and treatment of posttraumatic stress disorder:Methodology and development process. Journal of Traumatic Stress, 32, 475-483. https://doi.org/10.1002/jts.22421

Becker, C. B., Zayfert, C. \& Anderson, E. (2004). A survey of psychologists' attitudes towards and utilization of exposure therapy for PTSD. Behaviour Research and Therapy, 42, $277-292$. https://doi.org/10.1016/S0005-7967(03)00138-4

Chen, L. P., Murad, M. H., Paras, M. L., Colbenson, K. M., Sattler, A. L., Goranson, E. N. et al. (2010). Sexual abuse and lifetime diagnosis of psychiatric disorders: Systematic review and meta-analysis. Mayo Clinic Proceedings, 85, 618-629. https://doi. org/10.4065/mcp.2009.0583

Chorpita, B. F. \& Daleiden, E. L. (2014). Structuring the collaboration of science and service in pursuit of a shared vision. Journal of Clinical Child \& Adolescent Psychology: The Official Journal for the Society of Clinical Child and Adolescent Psychology, 43, 323 - 338. https://doi.org/10.1080/15374416.2013.828297

Clemens, V., Huber-Lang, M., Plener, P. L., Brähler, E., Brown, R. C. \& Fegert, J. M. (2018). Association of child maltreatment subtypes and long-term physical health in a German representative sample. European Journal of Psychotraumatology, 9, 1510278. https://doi.org/10.1080/20008198.2018.1510278

Courtois, C. A. \& Brown, L. S. (2019). Guideline orthodoxy and resulting limitations of the American Psychological Association's clinical practice guideline for the treatment of PTSD in adults. Psychotherapy, 56, 329-339. http://dx.doi.org/10.1037/ pst0000239

Cohen, J. A., Mannarino, A. P., Deblinger, E. \& Goldbeck L. (2009). Traumafokussierte kognitive Verhaltenstherapie bei Kindern und Jugendlichen. Heidelberg: Springer.

De Jongh, A. D., Resick, P. A., Zoellner, L. A., Van Minnen, A., Lee, C. W., Monson, C. M. et al. (2016). Critical analysis of the current treatment guidelines for complex PTSD in adults. Depression and Anxiety, 33, 359 - 369. https://doi.org/10.1002/da.22469

Department of Veterans Affairs and Department of Defense (VA/ DoD). (2017). VA/DoD clinical practice guideline for the management of posttraumatic stress disorder and acute stress disorder. Verfügbar unter https://www.healthquality.va.gov/guidelines/ MH/ptsd/VADoDPTSDCPGFinal012418.pdf

Farrell, N. R., Deacon, B. J., Kemp, J. J., Dixon, L. J. \& Sy, J. T. (2013). Do negative beliefs about exposure therapy cause its suboptimal delivery? An experimental investigation. Journal of Anxiety Disorders, 27, 763-771. https://doi.org/10.1016/j.janx dis.2013.03.007

Fegert, J. M., Rassenhofer, M., Schneider, T., Sproeber, N. \& Seitz, A. (2013). Sexueller Kindesmissbrauch - Zeugnisse, Botschaften, Konsequenzen. Weinheim: Beltz Juventa.

Ferrara, P., Corsello, G., Basile, M. C., Nigri, L., Campanozzi, A., Ehrich, J. \& Pettoello-Mantovani, M. (2015). The economic burden of child maltreatment in high income countries. The Journal of Pediatrics, 167, 1457 -1459. https://doi.org/10.1016/j.jpeds. 2015.09.044

Forbes, D., Bisson, J. I., Monson, C. M. \& Berliner, L. (2020). Effective treatments for PTSD. New York: Guilford Publications.

Forbes, D., Creamer, M., Bisson, J. I., Cohen, J. A., Crow, B. E., Foa, E. B. et al. (2010). A guide to guidelines for the treatment of PTSD and related conditions. Journal of Traumatic Stress, 23 , 537 - 552. https://doi.org/10.1002/jts.20565

Ganser, H. G., Münzer, A., Plener, P. L., Witt, A. \& Goldbeck, L. (2016). Children and adolescents after child abuse and neglect: Do they receive appropriate treatment? Bundesgesundheitsblatt - Gesundheitsforschung - Gesundheitsschutz, 59, $803-$ 810. https://doi.org/10.1007/s00103-016-2351-6

Hamblen, J. L., Norman, S. B., Sonis, J. H., Phelps, A. J., Bisson, J. I., Nunes, V. D. et al. (2019). A guide to guidelines for the treatment of posttraumatic stress disorder in adults: An update. Psychotherapy, 56, 359 - 373. https://doi.org/10.1037/pst0000231

Henning, J. A. \& Brand, B. L. (2019). Implications of the American Psychological Association's posttraumatic stress disorder treatment guideline for trauma education and training. Psychotherapy, 56, 422 - 430. https://doi.org/10.1037/pst0000237

Hipol, L. J. \& Deacon, B. J. (2013). Dissemination of evidence-based practices for anxiety disorders in Wyoming: A survey of practicing psychotherapists. Behavior Modification, 37, $170-$ 188. https://doi.org/10.1177/0145445512458794

Institute of Medicine (IOM). (2011). Clinical practice guidelines we can trust. Washington, DC: The National Academies Press.

ISTSS Guidelines Committee. (2019). ISTSS PTSD prevention and treatment guidelines methodology and recommendations. Verfügbar unter: https://istss.org/getattachment/Treating-Trau ma/New-ISTSS-Prevention-and-Treatment-Guidelines/ ISTSS_PreventionTreatmentGuidelines_FNL-March-19-2019. pdf.aspx

ISTSS Guidelines Committee. (n.d.). ISTSS guidelines position paper on complex PTSD in children and adolescents. Verfügbar unter: https://istss.org/getattachment/Treating-Trauma/NewISTSS-Prevention-and-Treatment-Guidelines/ISTSS_CPTSDPosition-Paper-(Child_Adol)_FNL.pdf.aspx

Landolt, M. A., Schnyder, U., Maier, T., Schoenbucher, V. \& MohlerKuo, M. (2013). Trauma exposure and posttraumatic stress disorder in adolescents: A national survey in Switzerland. Journal of Traumatic Stress, 26, 209-216. https://doi.org/10. 1002/jts. 21794

Lilienfeld, S. O., Ritschel, L. A., Lynn, S. J., Cautin, R. L. \& Latzman, R. D. (2013). Why many clinical psychologists are resistant to evidence-based practice: Root causes and constructive remedies. Clinical Psychology Review, 33, 883 -900. https://doi.org/ 10.1016/j.cpr.2012.09.008 
Meyer, J. M., Farrell, N. R., Kemp, J. J., Blakey, S. M. \& Deacon, B. J. (2014). Why do clinicians exclude anxious clients from exposure therapy? BehaviourResearch and Therapy, 54, 49-53. https://doi.org/10.1016/j.brat.2014.01.004

Münzer, A., Fegert, J. M. \& Goldbeck, L. (2015). Traumaanamnese und posttraumatische Stresssymptomatik in einer kinder- und jugendpsychiatrischen Inanspruchnahmepopulation. Psychiatrische Praxis, 42(2), 96 - 101. https://doi.org/10.1055/s-00331360058

National Institute for Health and Care Excellence. (2018). Posttraumatic stress disorder (NICE guideline NG116): [B] Evidence reviews for psychological, psychosocial and other non-pharmacological interventions for the treatment of PTSD in children and young people. Verfügbar unter https://www.nice.org.uk/gui dance/ng116/resources/posttraumatic-stress-disorder-pdf66141601777861

Nocon, A., Eberle-Sejari, R., Unterhitzenberger J. \& Rosner, R. (2017). The effectiveness of psychosocial interventions in young war-traumatized refugees: Systematic review and meta-analysis. European Journal of Psychotraumatology, 8, Article 1388709. https://doi.org/10.1080/20008198.2017.1388709

Norcross, J. C. \& Wampold, B. E. (2019). Relationships and responsiveness in the psychological treatment of trauma: The tragedy of the APA Clinical Practice Guideline. Psychotherapy, 56, 391 - 399. https://doi.org/10.1037/pst0000228

Phoenix Australia Centre for Posttraumatic Mental Health. (2020). Australian guidelines for the prevention and treatment of stress, disorder, posttraumatic stress disorder and complex PTSD. Verfügbar unter https://www.phoenixaustralia.org/wp-con tent/uploads/2020/11/3.-PTSD-Guidelines-Executive-summa ry.pdf

Rosner, R., Gutermann, J., Landolt, M. A., Plener, P. \& Steil, R. (2019). Diagnose und Behandlung der PTBS bei Kindern und Jugendlichen. In I. Schäfer, U. Gast, A. Hofmann, C. Knaevelsrud \& A. Lampe (Hrsg.), S3-Leitline Posttraumatische Belastungsstörung (S. 59-82). Berlin: Springer.

Rosner, R., Barke, A., Albrecht, B., Christiansen, H., Ebert, D. D., Lechner-Meichsner, F. et al. (2020). BEST FOR CAN-bringing empirically supported treatments to children and adolescents after child abuse and neglect: Study protocol. European Journal of Psychotraumatology, 11, 1837531. https://doi.org/10.1080/ 20008198.2020.1837531

Schäfer, I., Gast, U., Hofmann, A., Knaevelsrud, C., Lampe, A., Liebermann, P. et al. (2019). S3-Leitlinie Posttraumatische Belastungsstörung. Berlin: Springer.

Schauer, M., Neuner, F. \& Elbert, T. (2017). Narrative exposure therapy for children and adolescents (KIDNET). In M. A. Landolt, M. Cloitre \& U. Schnyder (Eds.), Evidence-based treatments for trauma related disorders in children and adolescents (pp. $97-$ 273). Cham: Springer International Publishing. https://doi.org/ 10.1007/978-3-319-46138-0_13

Stauffer, A., Petermann, F., Metzner, F. \& Vasileva, M. (2020). Behandlungsmöglichkeiten für Vorschulkinder mit traumatischen Erfahrungen: Eine systematische Literaturübersicht. Zeitschrift für Psychiatrie, Psychologie und Psychotherapie, 68, 33-44. https://doi.org/10.1024/1661-4747/a000402

Shapiro, F., Wesselmann, D. \& Mevissen, L. (2017). Eye movement desensitization and reprocessing therapy (EMDR). In M. A. Landolt, M. Cloitre \& U. Schnyder (Eds.), Evidence-based treatments for trauma related disorders in children and adolescents (pp. 97 -273). Cham: Springer International Publishing. https:// doi.org/10.1007/978-3-319-46138-0_13

Sholomskas, D. E., Syracuse-Siewert, G., Rounsaville, B. J., Ball, S. A., Nuro, K. F. \& Carroll, K. M. (2005). We don't train in vain: A dissemination trial of three strategies of training clinicians in cognitivebehavioral therapy. Journal of Consulting and Clinical Psychology, 73(1), 106 - 115. https://doi.org/10.1037/0022-006X.73.1.106

Silver, K. E. \& Levant, R. F. (2019). An appraisal of the American Psychological Association's Clinical Practice Guideline for the treatment of posttraumatic stress disorder. Psychotherapy, 56, 347-358. https://doi.org/10.1037/pst0000230

Thordardottir, E. B., Valdimarsdottir, U. A., Hansdottir, I., Hauksdóttir, A., Dyregrov, A., Shipherd, J. C. et al. (2016). Sixteenyear follow-up of childhood avalanche survivors. European Journal of Psychotraumatology, 7, 30995. https://doi.org/10. 3402/ejpt.v7.30995

Unterhitzenberger, J., Wintersohl, S., Lang, M., König, J. \& Rosner, R. (2019). Providing manualized individual trauma-focused CBT to unaccompanied refugee minors with uncertain residence status: A pilot study. Child and Adolescent Psychiatry and Mental Health, 13(1), 1-10. https://doi.org/10.1186/s13034019-0282-3

van Minnen, A., Hendriks, L. \& Olff, M. (2010). When do trauma experts choose exposure therapy for PTSD patients? A controlled study of therapist and patient factors. Behaviour Research and Therapy, 48, 312-320. https://doi.org/10.1016/j.brat. 2009.12.003

Whiteside, S. P., Deacon, B. J., Benito, K. \& Stewart, E. (2016). Factors associated with practitioners' use of exposure therapy for childhood anxiety disorders. Journal of Anxiety Disorders, 40, 29 - 36. https://doi.org/10.1016/j.janxdis.2016.04.001

Witt, A., Brown, R., Plener, P. L., Brähler, E., Fegert, J. M. \& Clemens, V. (2019). Kindesmisshandlung und deren Langzeitfolgen - Analyse einer repräsentativen deutschen Stichprobe. Zeitschrift für Psychiatrie, Psychologie und Psychotherapie, 67, 100 - 111. https://doi.org/10.1024/1661-4747/a000378

World Health Organization (WHO).(1992). The ICD-10 classification of mental and behavioural disorders: clinical descriptions and diagnostic guidelines. Geneva: WHO.

World Health Organization (WHO). (2018). ICD-11 for Mortality and Morbidity Statistics. Verfügbar unter: https://icd.who.int/brow se11/l-m/en

\section{Förderung}

Dieser Artikel ist im Rahmen des vom Bundesministerium für Bildung und Forschung (BMBF) geförderten Projektes „BESTFORCAN - Empirisch basierte Behandlung für Kinder und Jugendliche als Opfer von Missbrauch und Vernachlässigung zugänglich machen“ (FKZ: 01KR1804 A) entstanden.

Open Access-Veröffentlichung ermöglicht durch die Universitätsbibliothek Johann Christian Senckenberg.

\section{Apl. Prof. Dr. Regina Steil}

Goethe Universität Frankfurt

Abteilung Klinische Psychologie und Psychotherapie

Varrentrappstraße $40-42$

60486 Frankfurt am Main

steil@psych.uni-frankfurt.de 